\title{
Efectividad de la ventilación mecánica no invasiva en pacientes posextubados con enfermedad pulmonar obstructiva crónica
}

\section{Effectiveness of non-invasive mechanical ventilation in post-tube patients with chronic obstructive pulmonary disease.}

Carlos Alberto Molina Cárdenas ${ }^{1}$, Karen Lissette Sánchez Pincay ${ }^{2}$, Andreina Beatriz Borbor Cabrera. ${ }^{3}$, Celia Brunilda Bustamante Valencia. ${ }^{4}$

Recibido: 05-02-2020 / Revisado: 15-03-2020 /Aceptado: 04-04-2020/ Publicado: 05-06-2019

DOI: https://doi.org/10.33262/anatomiadigital.v3i2.1..1259

\begin{abstract}
Non-Invasive Mechanical Ventilation (NIMV) in post-tubed patients provides mechanical ventilatory support, without the use of an artificial airway, avoiding complications from prolonged use of Mechanical Ventilation, reducing the hospital stay of the Intensive Care Unit. To determine the effectiveness of non-invasive mechanical ventilation after its establishment in chronic obstructive pulmonary disease that affects the improvement of the quality of life of posttubal patients in the Intensive Care Unit of
\end{abstract}

\section{Resumen}

La Ventilación Mecánica No Invasiva (VMNI) en pacientes posextubados entrega un soporte ventilatorio mecánico, sin la utilización de una vía aérea artificial evitando complicaciones por el uso prolongado de la Ventilación Mecánica, disminuyendo la estancia hospitalaria de la Unidad de Cuidados Intensivos. Determinar la efectividad de la ventilación mecánica no invasiva posterior a su instauración en la enfermedad pulmonar obstructiva crónica que incide en la mejoría de la calidad de vida de los pacientes posextubados de la Unidad de Cuidados

\footnotetext{
${ }^{1}$ Universidad Estatal de Milagro. Maestría en Salud Pública, Milagro, Ecuador, Hospital de Especialidades Dr. Teodoro Maldonado Carbo, Guayaquil Ecuador, albertocar190@hotmail.com, https://orcid.org/0000-0003-26776491

${ }^{2}$ Hospital de Especialidades Dr. Teodoro Maldonado Carbo, Guayaquil, Ecuador, karensanchez-92@hotmail.com, https://orcid.org/0000-0002-3049-983X,

${ }^{3}$ Hospital de Especialidades Dr. Teodoro Maldonado Carbo, Guayaquil, Ecuador, andreina-borbor@hotmail.com, https://orcid.org/0000-0003-2769-4308,

${ }^{4}$ Hospital de Especialidades Dr. Teodoro Maldonado Carbo, Guayaquil, Ecuador, celia.bustamantev@ug.edu.ec, https://orcid.org/0000-0001-7307-5351
} 
the Teodoro Maldonado Carbo Hospital in the city of Guayaquil. Quantitative, nonexperimental, cross-sectional, descriptive study; with a convenience sample directed at adult patients inside the Intensive Care Unit (ICU), the population was 100 cases and the sample type was non-probability for convenience, selecting the population that met the characteristics for the research, taken from the clinical records of the AS400 system, corresponding to the period from the month of January 2018 to January 2019, an observation sheet was made that consisted of 16 items which was validated by judgments of specialists and health experts qualifying validity, relevance and coherence. The results on the management of patients diagnosed with COPD and undergoing Non-Invasive Mechanical Ventilation post-incubation have been shown to overcome respiratory failure, reducing muscle fatigue due to excessive use of accessory muscles; In this way, it improves arterial $\mathrm{PH}, \mathrm{PO} 2$, and $\mathrm{PCO} 2$ levels, reducing the days of hospitalization in the ICU, pneumonia associated with invasive mechanical ventilation and reintubation, allowing a reduction in the days of hospital stay. Non-invasive mechanical ventilation applied in the early period to extubation in patients with a diagnosis of exacerbated COPD is useful to improve the results in this disease by considerably reducing the incidence of pneumonia associated with mechanical ventilation, reintubation, and therefore the total mechanical ventilation time in the ICU.
Intensivos del Hospital Teodoro Maldonado Carbo de la ciudad de Guayaquil. Estudio cuantitativo, no experimental, de corte transversal, descriptivo; con una muestra por conveniencia dirigida a los pacientes adultos al interior de la Unidad de Cuidado Intensivo (UCI), la población fue de 100 casos y el tipo de muestra fue no probabilística por conveniencia, seleccionando a la población que cumplieron con las características para la investigación, tomados de las historias clínicas del sistema AS400, correspondiente al periodo del mes de Enero del 2018 hasta Enero del 2019, se realizó una ficha de observación que constó con 16 ítems el cual fue validado por juicios de especialistas y expertos en salud calificando validez, pertinencia y coherencia. Los resultados sobre el manejo de los pacientes con diagnóstico de EPOC y sometidos a Ventilación Mecánica No Invasiva posextubacion ha demostrado superar la insuficiencia respiratoria, reduciendo la fatiga muscular por el uso excesivo de la musculatura accesoria; de esta manera mejora los niveles de $\mathrm{PH}, \mathrm{PO} 2, \mathrm{PCO} 2$ arterial, reduciendo los días de hospitalización en UCI, neumonía asociada a ventilación mecánica invasiva y la reintubacion permitiendo la reducción de los días de estancia hospitalaria. La Ventilación Mecánica No Invasiva aplicada en el período temprano a la extubación en los pacientes con diagnóstico de EPOC exacerbada es útil para mejorar la los resultados en esta enfermedad al disminuir considerablemente la incidencia de neumonía asociada a la ventilación mecánica, la reintubación y por tanto el tiempo total de ventilación mecánica en la UCI. 
Keywords: Non-invasive mechanical ventilation, chronic obstructive pulmonary disease, Intensive care unit, acute respiratory failure
Palabras Claves: Ventilación mecánica no invasiva, enfermedad pulmonar obstructiva crónica, Unidad de cuidados intensivos, insuficiencia respiratoria aguda.

\section{Introducción}

El progreso alcanzado por la Salud Pública contemporánea es expresión de la constante preocupación acontecida a nivel mundial por mejorar las condiciones de vida de las poblaciones; implicación devenida de la atención a la diversidad de determinantes sociales que imperan en los distintos contextos.

Dicho logro gestionó el tránsito de la visión positivista del enfoque biomédico; concepción unipolar que busca explicación a las causas que irrumpen el proceso salud-enfermedad, en las conductas individuales y/o rasgos de la biología humana, por el alcance de la tendencia hegemónica del modelo de atención integral de salud al individuo, la familia y la comunidad; paradigma salubrista que guía el cuidado con un enfoque centrado en la promoción de la salud y la prevención de las enfermedades.

Como parte de esa configuración se realza la necesidad de desarrollar acciones preventivas, dirigidas a la disminución de la morbimortalidad por enfermedades no trasmisibles (ENT), las que generan una alta carga para los sistemas sanitarios por factores evitables. Al respecto, la Organización Mundial de la Salud (OMS) reporta que el 71\% de los decesos que se producen cada año, obedecen a esa causa. (Organización Mundial de la Salud. Enfermedades No Transmisibles. Datos y cifras. Ginebra, 2018.)

Engrosando el abanico de ENT se encuentra la Enfermedad Pulmonar Obstructiva Crónica (EPOC), patología enmarcada entre las entidades respiratorias responsables del 3,9 de los 41 millones de fallecidos anuales por causas crónicas.

La EPOC es una enfermedad crónica inflamatoria de los pulmones que obstruye el flujo de aire desde los pulmones. Según estimaciones de la OMS, se calcula que para el 2030 dicha enfermedad constituirá la cuarta causa de muerte en todo el mundo. (Organización Mundial de la Salud. Departamento de Enfermedades Crónicas y Promoción de la Salud Enfermedad pulmonar obstructiva crónica (EPOC). Ginebra, 2018.)

La exacerbación de la EPOC provoca el $20 \%$ de los motivos de consulta en los servicios de urgencias médicas y se estima que aproximadamente el $25 \%$ de estos pacientes presentan en la gasometría arterial acidosis respiratoria, el cual es indicativo de falla respiratoria. El tratamiento de esta complicación se basa en el apoyo convencional farmacológico y de soporte respiratorio mediante el uso de ventilación mecánica no invasiva (VMNI). (Dra. Caridad Ortiz Zamora, 2015) 
La evidencia terapéutica como beneficios de la VMNI en el tratamiento de la insuficiencia respiratoria aguda de los pacientes con EPOC ha sido ampliamente demostrada por la literatura científica, impacto confirmado por el alto grado de recomendación de esa terapia y por el progresivo incremento de su uso por parte de los especialistas de la salud en el área de Cuidados Intensivos. (Grupo de Trabajo de GesEPOC, 2012)

Para autores como Puga, el éxito de la VMNI responde a que la misma permite la administración de soporte ventilatorio no invasivo sin la colocación de una vía aérea artificial, es decir mediante la colocación de un tubo endotraqueal, facilitando de esta manera la oxigenación hacia los pulmones y permitiendo un adecuado intercambio gaseoso. (Superior de Medicina Militar et al., 2006)

En su lugar se emplea una máscara naso-bucal para ventilar al paciente. Los efectos beneficiosos de este tipo de tratamiento se aprecian en la disminución del trabajo respiratorio, la mejoría de la ventilación alveolo-pulmonar y la reducción de la frecuencia de intubación o reintubación; por lo que se recomienda en la insuficiencia respiratoria aguda (IRA) y como estrategia de rescate en pacientes con EPOC postextubados, pues permite reducir infecciones nosocomiales, como lo son en gran escala las neumonías asociadas a la ventilación mecánica invasiva y de esta manera se acorta la estadía hospitalaria por esa causa.

La tendencia contemporánea centra su atención en mantener conectado al paciente el menor tiempo posible al ventilador mecánico, lo que significa que este debe ser retirado de ese tratamiento de forma inmediata y oportuna; a este proceso se le llama destete, de esta manera se evitan complicaciones causadas por la ventilación invasiva a largos periodos

El cuidado del paciente con VMNI representa un reto para el equipo de salud encargado de brindar atención integral a pacientes que sufren de afecciones cardio-respiratorias o respiratorias agudas o crónicas, en el que el Terapista Respiratorio asume un rol importante en la prevención temprana del deterioro respiratorio progresivo. (Martín-González, et al., 2016)

Los beneficios del empleo de la VMNI avalan su uso en aquellos pacientes tributarios de esta, información no documentada por el departamento de Terapia Respiratoria del Hospital Teodoro Maldonado Carbo de la ciudad de Guayaquil, circunstancia que motivó al investigador del estudio que se presenta a indagar sobre esa problemática, con el objetivo de describir esa realidad contextual, desde el impacto positivo que el enfoque preventivo de la utilización de la misma genera para el paciente con EPOC, su familia, el sistema de salud y la sociedad en su conjunto. (Ruiz, et al., 2018)

\section{Materiales y Métodos}

Se realizó un estudio retrospectivo, observacional descriptivo, de corte longitudinal; en el que la población de estudio estuvo constituida por los 100 pacientes hospitalizados en la Unidad de 
Cuidados Intensivos (UCI) del Hospital De Especialidades Teodoro Maldonado Carbo de la ciudad de Guayaquil, durante el período comprendido de enero 2018 a enero 2019; de los cuales, siguiendo un muestreo no probabilístico del tipo intencional o por juicio, ${ }^{(9)}$ se seleccionaron aquellas correspondiente a individuos con edades de 35 a 66 años, de ambos sexos, diagnosticados con EPOC y tratados con VMNI por insuficiencia respiratoria aguda (100 individuos).

Los datos fueron obtenidos mediante la técnica de observación, para lo cual se empleó una ficha de observación del paciente, la que fue diseñada por el autor del estudio que se presenta y validada por criterio de expertos. Proceso que permitió eliminar aspectos irrelevantes, incorporar aquellos considerados como imprescindibles y modificar los que lo requirieron. (Arturo \& Trápaga, s. f.)

Con la finalidad de obtener elementos de valor sobre los ítems incluidos en el instrumento denominado ficha de observación, el mismo se aplicó vía correo electrónico a seis profesionales con experiencia laboral en la atención a pacientes ventilados en UCI, con formación en el área de Medicina Critica y metodólogos con grado académico de maestría en investigación y docencia universitaria.

Los participantes evaluaron satisfactoriamente el instrumento conformado por tres dimensiones:

- La primera incluyó aspectos de orden demográficos: edad, género, procedencia, nivel de instrucción y ocupación.

- La segunda criterios de la valoración del paciente al inicio de la VMNI: diagnóstico médico de ingreso, valoración clínica, niveles de gases arteriales, sintomatología antes y después del tratamiento ventilatorio no invasivo.

- La tercera exploró elementos relativos a la intervención basada en el soporte ventilatorio no invasivo: sintomatología del paciente durante al tratamiento con VMNI y al momento de la extubación; modalidad de ventilación por presión positiva de vía aérea (CPAP) o por presión positiva de vía aérea en dos niveles (BIPAP), parámetros vitales, interface, la duración de la ventilación y la necesidad de ventilación mecánica controlada (VMC) ante fracaso de la no invasiva.

En relación con los contenidos explorados, el 58,5\% de los participantes en la validación plantearon estar de acuerdo con lo indagado. El 27,67\% sugirieron modificaciones a tres o más formulaciones y el 13,83\% a menos de esa cantidad. El instrumento validado permitió agrupar las variables de interés investigativo, las que luego fueron organizadas en una base de IBM SPSS Statistics 22.

Se tomaron en consideración los elementos éticos de la investigación científica, al respetar la autodeterminación de los sujetos a participar en el estudio y solicitar las debidas autorizaciones. 
La disposición de los resultados se hizo de manera tal que reflejara la no maleficencia o malevolencia al exponer los mismos.

\section{Resultados}

Al explorar la evolución de los pacientes con el empleo de la VMNI en relación con el tiempo de aplicación de esa terapéutica, la información observada evidencia que, de forma mayoritaria, el 85,8 de las personas incluidas en la muestra de estudio $(n=100)$ evolucionaron favorablemente.

El empleo de la VMNI demostró beneficios para los pacientes tratados con esa terapéutica, los que en las primeras dos horas de tratamiento alcanzaron mejoría $\mathrm{PH}, \mathrm{PaCO} 2$ y $\mathrm{PO} 2$; valores constituidos en la gasometría arterial; indicador predictor de éxito para valorar la efectividad de ese tipo de ventilación no invasiva y de esta manera evitar la reintubación endotraqueal del paciente posextubado, elemento que se acorta la estadía hospitalaria.

De igual forma, el 84\% de los pacientes entre 35 a 65 años mostraron mejoría con la aplicación de la VMNI posextubacion; de los cuales, sólo el 14\% requirió re-intubación endotraqueal. En relación con la saturación de oxígeno, el $87 \%$ de los casos reportaron valores de $\mathrm{PaO} 2$ por encima de $100 \mathrm{mmHg}$, saturación mayor o igual del > 90\%.

Los pacientes sometidos a la VMNI como tratamiento de rescate, mejoraron considerablemente su trabajo respiratorio, evitando el uso de la musculatura accesoria permitiendo y evitando fatiga muscular; campos pulmonares permeables a la auscultación con ausencia de ruidos patológicos a la auscultación.

La demostración de la eficacia de la VMNI en el tratamiento de la insuficiencia respiratoria es amplia en el ámbito científico. Sus principales objetivos se enfocan en la posibilidad de evitar la intubación y reintubación endotraqueal, la ventilación mecánica invasiva y sus potenciales complicaciones, como por ejemplo neumonías asociadas a la ventilación mecánica. Además de lo citado, este tipo de terapéutica respiratoria permite reducir el trabajo respiratorio, corregir la hipoxemia y la acidosis respiratoria. Resultados que pueden variar en dependencia del tipo de insuficiencia respiratoria, la instauración oportuna, monitorización ventilatoria, estado clínico del paciente y comorbilidad asociada.

Autores como Brochard (2003) plantean que esta terapéutica es confortable para el paciente ya que le permite un mejor confort, por ejemplo, mejor comunicación con el personal de salud comer y beber, expectorar, sin requerimiento de sedación profunda y se preservan los mecanismos de defensa de la vía aérea superior.

Actualmente, esta terapéutica constituye el tratamiento de elección en la mayor parte de pacientes con fallo respiratorio agudo. Los buenos resultados obtenidos en las agudizaciones 
graves de la EPOC y su uso en pacientes posextubados, han extendido la terapéutica de la VMNI a otras unidades asistenciales; y patologías que cursan con insuficiencia respiratoria aguda, lo que hace que la misma sea considerada una intervención de primera línea en el manejo de estos pacientes. (Kalil et al., 2016)

Su uso precoz en la Unidad de Cuidados Intensivos (UCI) con fallo respiratorio, la administración temprana de soporte ventilatorio no invasivo con intervalos de descanso se asocia a una mejoría significativa de los parámetros fisiológicos, sintomatología y la calidad de vida del paciente, acortando su estadía hospitalaria. (Mehta S, Hill NS.)

Investigadores dedicados al estudio de esta temática y a la demostración de sus resultados, han identificado la existencia de factores pronósticos de éxito para la VMNI; entre los que se encuentran la edad, la presencia de poca cantidad de secreciones respiratorias, alta puntuación en la evaluación de neurológica y bajo índice de gravedad, esta última determinada mediante la aplicación de la Acute Physiology and Chronic Health Evaluation II (APACHE II), con resultados menores de 25 puntos, adecuada sincronía del paciente con el ventilador con escaso escape a través de la interface y una correcta adaptación de este al equipo. (Mehta S, Hill NS.); (Benito \& Epstein, 2002)

Formando parte de los criterios pronósticos antes dichos se encuentran la acidemia de moderada intensidad ( $\mathrm{pH}$ mayor de 7,10 y menor de 7,35) y las cifras de presión arterial de dióxido de carbono (PaCO2) entre 45 y $92 \mathrm{mmHg}$ (hipercapnia discreta). Indicadores que al decir de autores como Rialp, Avarro, Galeas y Navarro expresan la evolución del paciente, la que puede apreciarse dos horas después de iniciada este tipo de terapéutica.(Cervera, et al., 2014; (Reyes, et al., 2020);(Muñoz Bono J, Curiel Balsera E, Galeas López J, 2011;Betancourt-Reyes, 2018)

\section{Conclusiones}

- La Ventilación Mecánica No Invasiva aplicada en el período temprano a la extubación en los pacientes con EPOC exacerbada es útil para mejorar los resultados en esta enfermedad al disminuir considerablemente la incidencia de neumonía asociada a la ventilación mecánica, la reintubación y, por tanto, el tiempo total de ventilación mecánica en la UCI.

- El paciente con exacerbación de EPOC experimenta un conjunto de trastornos fisiopatológicos, la cual aumentan considerablemente la morbimortalidad y al mismo tiempo alarga la estancia hospitalaria.

- En cuanto la VMNI con modalidad BIPAP aplicada en el período inmediato a la extubación en los pacientes con EPOC exacerbada es útil para mejorar los resultados en esta enfermedad al disminuir la incidencia de neumonía asociada a la ventilación mecánica, la reintubación y, por tanto, el tiempo total de ventilación, todo esto conlleva 
a una disminución de la mortalidad por esta enfermedad que tan problemática ha sido en los últimos años en el entorno de los cuidados intensivos.

- La tolerancia a la mascarilla no invasiva conlleva a un adecuado confort ventilador / paciente garantizando su sincronía respiratoria y disminuyendo el trabajo ventilatorio; de esta manera la asistencia ventilatoria no invasiva se ha constituido en una valiosa herramienta terapéutica en este contexto, y permite solucionar exitosamente hasta el ochenta por ciento de estos casos, reduciendo costos, estadía, complicaciones y mortalidad.

\section{Referencias Bibliográfica}

Arturo, J., \& Trápaga, B. (s. f.). Validez de contenido por juicio de expertos: propuesta de una herramienta virtual, 27, 42-53.

Benito, S., \& Epstein, S. K. (2002). Esteban A, Anzueto A, Frutos F, et al: Characteristics and outcomes in adult patients receiving mechanical ventilation: A 28-day international study. JAMA 287: 345-355, (January), 345-355. https://doi.org/10.1001/jama.287.3.345

Betancourt-Reyes, G. L. (2018). Efectividad de la ventilación ó n mecánica no invasiva en pacientes con insuficiencia respiratoria aguda Effectiveness of non - invasive mechanical ventilation in patients with acute respiratory failure, 43 .

Brochard, L. (2003). Mechanical ventilation: invasive versus noninvasive, 31-37. https://doi.org/10.1183/09031936.03.00050403

Cervera, G. R., Blanco, A. C., Aizcorreta, O. P., Parra, L., \& Semicyuc, G. De. (2014). Ventilación mecánica no invasiva en la enfermedad pulmonar obstructiva crónica y en el edema agudo de pulmón cardiogénico, 38(2).

Dra. Caridad Ortiz Zamora. (2015), 19(10), 1224-1231.

Grupo de Trabajo de GesEPOC. (2012). Guía de Práctica Clínica para el Diagnóstico y Tratamiento de Pacientes con Enfermedad Pulmonar Obstructiva Crónica ( EPOC ) Guía Española de la EPOC (GesEPOC ) Clinical Practice Guideline for the Diagnosis and Treatment of Patients with Chronic Obstru. Archivos de Bronconeumología, 48(Supl 1), 2-58.

Kalil, A. C., Metersky, M. L., Klompas, M., Muscedere, J., Sweeney, D. A., Palmer, L. B., ... Grady, N. P. O. (2016). Management of Adults With Hospital-acquired and Ventilatorassociated Pneumonia: 2016 Clinical Practice Guidelines by the Infectious Diseases Society of America and the American Thoracic Society, 63, 61-111. https://doi.org/10.1093/cid/ciw353

Martín-González, F., González-Robledo, J., Sánchez-Hernández, F., Moreno-García, M. N., \& Barreda-Mellado, I. (2016). Efectividad y predictores de fracaso de la ventilación mecánica no invasiva en la insuficiencia respiratoria aguda. Medicina Intensiva, 40(1), 917. https://doi.org/10.1016/j.medin.2015.01.007

Medisan 2013;17(5): 760. (2013), 17(5), 760-766. 
Organización Mundial de la Salud. Enfermedades No Transmisibles. Datos y cifras. Ginebra, 2018. Disponible en: https://www.who.int/es/news-room/factsheets/detail/noncommunicable-diseases.

Organización Mundial de la Salud. Departamento de Enfermedades Crónicas y Promoción de la Salud Enfermedad pulmonar obstructiva crónica (EPOC). Ginebra, 2018. Disponible en: https://www.who.int/respiratory/copd/es/

Reyes, B., Universitario, H., Argilagos, A. S., \& Intensivos, C. (2020). El auge de la ventilación mecánica no invasiva en el siglo XXI, 19(1), 19-21.

Ruiz, A. H., Iracema, R., Fernández, D., Rafael, G., \& Mustelier, A. (2018). Revista Habanera de Ciencias Médicas, 17(6), 885-895.

Sánchez-Hernández, F., Martín-González, F., Barreda-Mellado, I., González-Robledo, J., \& Moreno-García, M. N. (2015). Efectividad y predictores de fracaso de la ventilación mecánica no invasiva en la insuficiencia respiratoria aguda. Medicina Intensiva, 40(1), 917. https://doi.org/10.1016/j.medin.2015.01.007

Superior de Medicina Militar, I., Díaz Soto, L., Santiago Puga Torres, M., Palacios Pérez, H., García Valdés, R., \& Morejón Carbonell, D. (2006). Rev. Cubana Med Milit 2006;35(2) Ventilación no invasiva, 35(2), 1-9. Recuperado a partir de http://bvs.sld.cu/revistas/mil/vol35_2_06/mil08206.pdf 


\section{PARA CITAR EL ARTÍCULO INDEXADO.}

Molina Cárdenas, C. A., Sánchez Pincay, K. L., Borbor Cabrera, A. B., \& Bustamante Valencia, C. B. (2020). Efectividad de la ventilación mecánica no invasiva en pacientes posextubados con enfermedad pulmonar obstructiva crónica. Anatomía Digital, 3(2.1.), 32-41. https://doi.org/10.33262/anatomiadigital.v3i2.1.1259

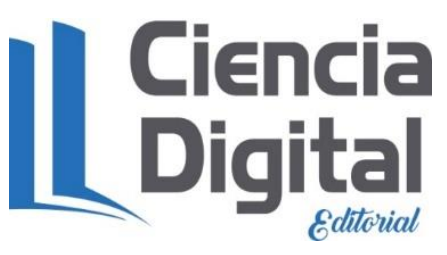

El artículo que se publica es de exclusiva responsabilidad de los autores y no necesariamente reflejan el pensamiento de la Revista Anatomía Digital.

El artículo queda en propiedad de la revista y, por tanto, su publicación parcial y/o total en otro medio tiene que ser autorizado por el director de la Revista Anatomía Digital.
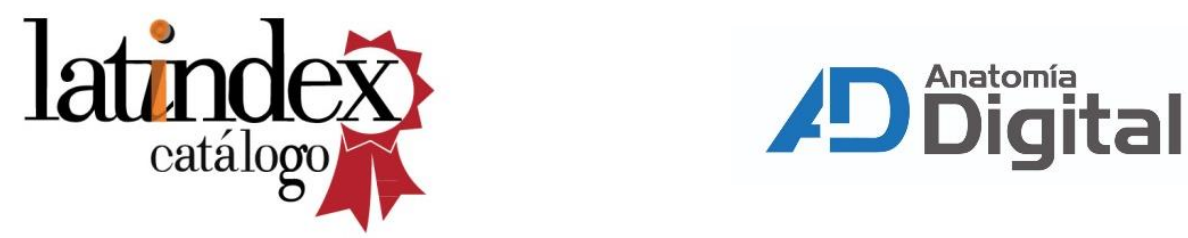